Case Report

\title{
Acute Facial Edema in a Patient with Systemic Lupus Erythematosus
}

\author{
Shiho Amano ${ }^{1}$, Ryuichi Ohta ${ }^{1, *}$ and Chiaki Sano ${ }^{2}$ (D) \\ 1 Community Care, Unnan City Hospital, Unnan 699-1221, Shimane, Japan; af5.swcot.gr@gmail.com \\ 2 Department of Community Medicine Management, Faculty of Medicine, Shimane University, \\ Izumo 693-8501, Shimane, Japan; sanochi@med.shimane-u.ac.jp \\ * Correspondence: ryuichiohta0120@gmail.com
}

check for

updates

Citation: Amano, S.; Ohta, R.; Sano, C. Acute Facial Edema in a Patient with Systemic Lupus Erythematosus. Reports 2021, 4, 22. https://doi.org/ $10.3390 /$ reports 4030022

Academic Editor: Toshio Hattori

Received: 13 July 2021

Accepted: 30 July 2021

Published: 2 August 2021

Publisher's Note: MDPI stays neutral with regard to jurisdictional claims in published maps and institutional affiliations.

\begin{abstract}
Allergies have been found to be associated with systemic lupus erythematosus (SLE). However, few reports have described angioedema occurring in elderly men with systemic lupus erythematosus. Herein, we report the case of an 85-year-old man who presented with angioedema with eosinophilia. The patient was initially thought to have a drug-induced allergy. The differentiation between allergic reactions caused by drugs and those caused by eosinophilia with SLE can be challenging. The effect of the withdrawal of the suspected culprit drug and allergic dermal findings can be key to differentiating the two conditions. SLE is prevalent among younger generations; hence, active immunity can induce various symptoms, including eosinophilia, which causes angioedema. Even older people with SLE can have a strong immune reaction, resulting in angioedema with eosinophilia. In cases of localized facial edema in elderly patients with SLE, it is critical to consider angioedema caused by eosinophilia as a differential diagnosis.
\end{abstract}

Keywords: angioedema; systemic lupus erythematosus; drug-induced allergy; eosinophilia; elderly patient

\section{Case}

Allergies have been found to be associated with systemic lupus erythematosus (SLE). However, there are few reports that describe angioedema occurring in elderly men with systemic lupus erythematosus. Herein, we report the case of an 85-year-old man who presented with angioedema with eosinophilia.

An 85-year-old man with a past medical history of SLE visited our hospital with a chief complaint of acute facial edema. Two years before the visit, he was diagnosed with SLE based on the finding of facial erythema, lymphocytopenia $(546 / \mu \mathrm{L})$, the presence of positive antinuclear antibody at a titer of 1:160, positive double-strand DNA antibody $(8 \mathrm{IU} / \mathrm{mL})$, and positive anti-cardiolipin antibody $(21.5 \mathrm{U} / \mathrm{mL})$. He had been treated with hydroxychloroquine ( $200 \mathrm{mg}$ /day) without exacerbation of SLE. Ten days before the visit, he started using clobetasol propionate ointment for pruritus, mild scalp edema, and erythema. Four days before coming to the hospital, the facial edema worsened rapidly; hence, he visited our hospital and was diagnosed with a drug-induced allergy due to clobetasol propionate ointment. However, the facial edema continued to worsen, even after the culprit drug was discontinued. Thus, the patient was admitted for further assessment and treatment. The patient did not give a positive history of usage of medicines that may cause angioedema, such as angiotensin-converting enzyme inhibitors. At the time of admission, his vital signs were stable. He had facial pitting edema with bilateral posterior cervical lymphadenopathy mainly on the lower eyelids (Figure 1). Edema was not noted anywhere else on the body, including the airway. An initial investigation revealed eosinophilia (eosinophil count, 6396/ $\mu \mathrm{L}$ (normal rage, 70 to $450 / \mu \mathrm{L}$ )) and elevated $\operatorname{IgE}$ levels (1044 IU / mL (normal range, less than 173)). His renal function and complement levels were normal. He was diagnosed with angioedema due to the eosinophilia. As 
the symptom worsened after admission, we treated the patient with $1 \mathrm{mg} / \mathrm{kg} /$ day of prednisolone (PSL), and the symptom improved (Figure 2).

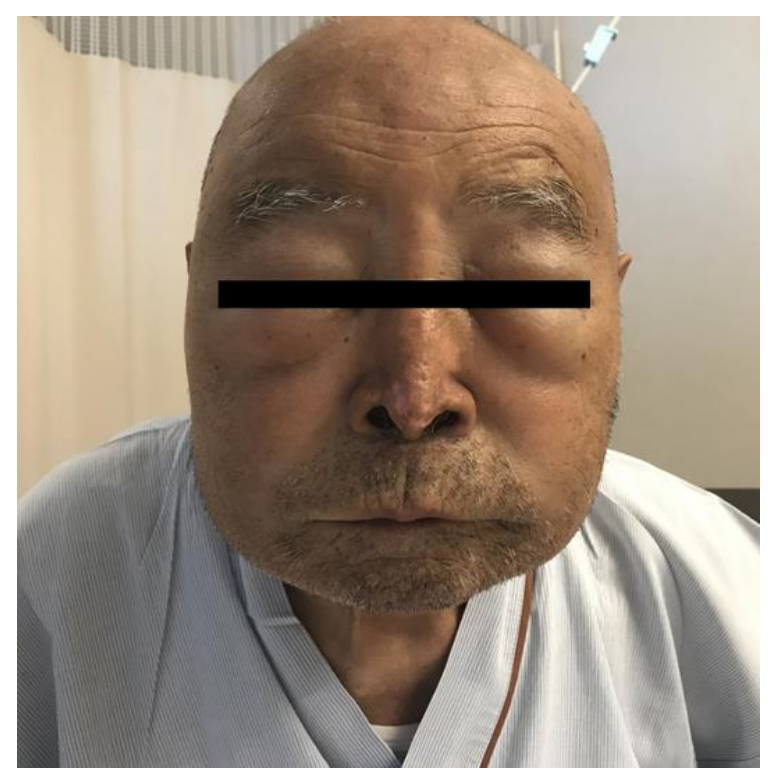

Figure 1. Facial pitting edema mainly on the eyelids at time of hospital admission.

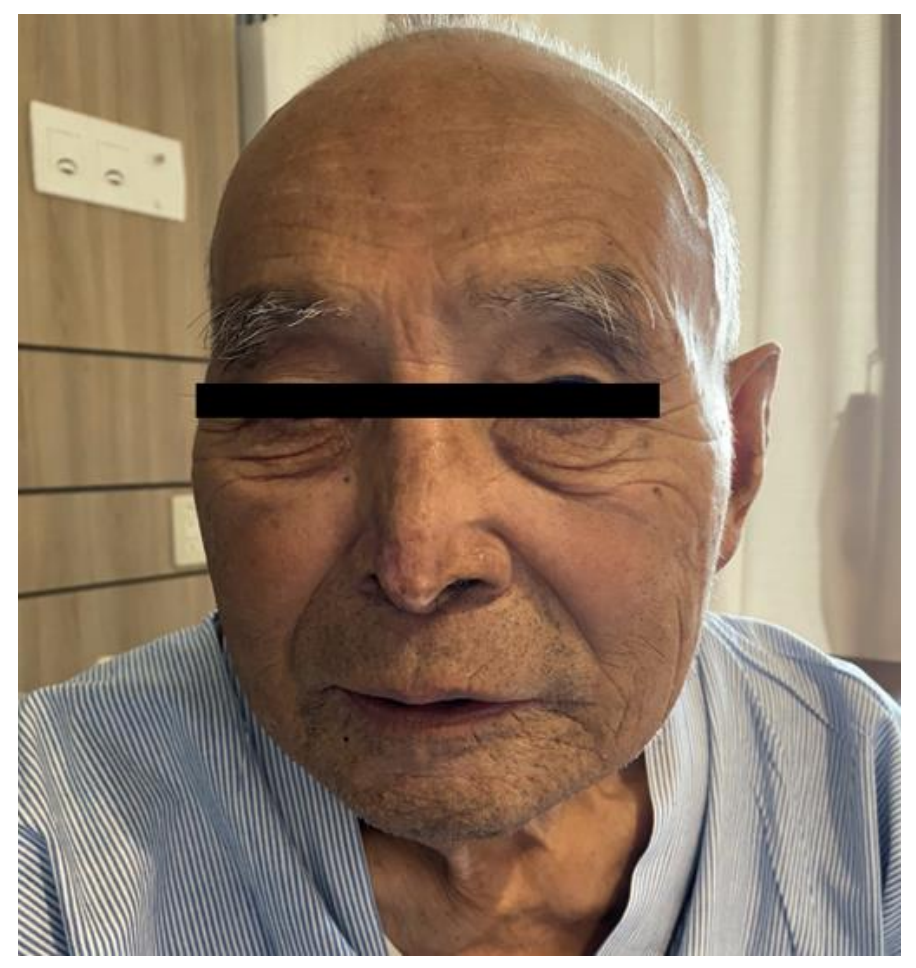

Figure 2. After treatment with $1 \mathrm{mg} / \mathrm{kg} /$ day of prednisolone (PSL).

After a 1-week treatment, we decreased the dose to $0.5 \mathrm{mg} / \mathrm{kg} /$ day. His symptoms did not worsen; thus, the PSL dosage was gradually reduced to $10 \mathrm{mg} /$ week. After the treatment, the eosinophil count and IgE level were within the normal range.

\section{Discussion}

This case is a rare presentation of facial edema and eosinophilia with SLE. Based on a previous report, the prevalence of SLE among men is rare [1], and there are very few 
case reports regarding facial edema with SLE among men [2]. The relationship between allergies and SLE is considered the cause of angioedema with the SLE in several studies [3,4]; allergies and SLE share several pathophysiological mechanisms [5]. It has been reported that dysregulation of the immune system occurs due to B-cell hyperactivity, leading to the production of immunoglobulins and autoantibodies [4]. In this case, the patient was initially suspected of having a drug-induced allergy. Differentiating allergic reactions caused by drugs from those caused by eosinophilia with SLE can be challenging. The effect of the withdrawal of the suspected culprit drug and allergic dermal findings can be key to differentiating between the two conditions [4].

In angioedema with SLE, eosinophil invasion is localized to the subdermal layer, which can be related to each of the conditions without allergic dermal expressions [6]. Furthermore, SLE is prevalent among younger generations; hence, active immunity can induce various symptoms, including eosinophilia, which causes angioedema. Considering that older people may not have a strong immunity, they rarely develop SLE [7]. However, our patient had angioedema and had previously been diagnosed with SLE. SLE may show the presence of strong immune reactions in affected individuals, causing several autoimmune reactions. Older people with SLE can have a strong immunity, resulting in angioedema with eosinophilia. In cases of localized facial edema in elderly patients with SLE, it is critical to consider angioedema caused by eosinophilia as a differential diagnosis. Notably, even if the sign is localized, systemic reactions should still be considered. Furthermore, even after discontinuing the use of the culprit drug, a pulse steroid therapy is required in severe cases [8]. Considering the possibility of developing severe reactions, early diagnosis and treatment are required.

Author Contributions: Conceptualization, S.A. and R.O.; methodology, R.O.; writing-original draft preparation, S.A. and R.O.; writing-review and editing, R.O.; supervision, C.S.; project administration, C.S.; funding acquisition, C.S. All authors have read and agreed to the published version of the manuscript.

Funding: This research received no external funding.

Institutional Review Board Statement: The ethical review and approval were waived for this study, due to the description of a single clinical case.

Informed Consent Statement: Informed consent was obtained from all subjects involved in the study.

Data Availability Statement: Not applicable.

Acknowledgments: We would like to thank this patient.

Conflicts of Interest: The authors declare no conflict of interest.

\section{References}

1. Kaul, A.; Gordon, C.; Crow, M.K.; Touma, Z.; Urowitz, M.B.; van Vollenhoven, R.; Ruiz-Irastorza, G.; Hughes, G. Systemic lupus erythematosus. Nat. Rev. Dis. Primers 2016, 2, 16039. [CrossRef]

2. Castro, L.A.; Davis, D.M.R.; Davis, M.D.P.; Bruce, A.J.; Pittelkow, M.R. Facial edema and crusted patches: A precursor to life-threatening acute systemic lupus erythematosus. J. Am. Acad. Dermatol. 2007, 56 (Suppl. 5), S126-S127. [CrossRef] [PubMed]

3. Hsiao, Y.P.; Tsai, J.D.; Muo, C.H.; Tsai, C.H.; Sung, F.C.; Liao, Y.T.; Chang, Y.J.; Yang, J.H. Atopic diseases and systemic lupus erythematosus: An epidemiological study of the risks and correlations. Int. J. Environ. Res. Public Health 2014, 11, 8112-8122. [CrossRef]

4. Luo, Y.; Fan, X.; Jiang, C.; Ramos-Rodriguez, A.; Wen, Y.; Zhang, J.; Huang, F.; Guan, X.; Xu, J. Systemic lupus erythematosus and angioedema: A cross-sectional study from the National Inpatient Sample. Arch. Rheumatol. 2019, 34, 301-307. [CrossRef]

5. Lamri, Y.; Charles, N. IgE in the Pathogenesis of SLE: From Pathogenic Role to Therapeutic Target. Antibodies 2020, 9, 69. [CrossRef] [PubMed]

6. Young, J.W.S.; Shear, N.H. Cutaneous drug reactions in the elderly. Drugs Aging 2017, 34, 655-672. [CrossRef]

7. Wozniacka, A.; Sysa-Jedrzejowska, S.; Robak, E.; Samochoki, Z.; Zak-Prelich, M. Allergic diseases, drug adverse reactions and total immunoglobulin E levels in lupus erythematosus patient. Mediat. Inflamm. 2003, 12, 95-99. [CrossRef] [PubMed]

8. Thong, B.Y.; Thumboo, J.; Howe, H.S.; Feng, P.H. Life-threatening angioedema in systemic lupus erythematosus. Lupus 2001, 10, 304-308. [CrossRef] 